\title{
XXI.
}

\section{Ein Beitrag zum Krankheitsbild der Chorea chronica progressiva.}

\author{
Von \\ Dr. R. Frotscher, \\ Oberarzt an der I,andes-Heil- und Pflegeanstalt Weilmünster, kreisärzllich geprüft.
}

Die Chorea chronica progressiva oder Chorea Huntington ist nach verschiedenen Richtungen hin eine noch recht unklare Krankheit. Nach Oppenheim (14) ist sie ein im ganzen seltenes Leiden, eine Krankheit sui generis, die scharf von dem bekannten Krankheitsbilde der Chorea minor zu trennen ist und die eine ausgesprochene Neigung besitzt, sich durch Generationen fortzuerben. 1872 ist sie zuerst von dem amerikanischen Arzt Huntington beschrieben worden, wurde aber lange Zeit nicht recht beachtet und erst im letzten Jahrzehnt wieder allgemein bekannt. Steyerthal (18) hat 1908 die Originalpublikation Huntingtons, die nur schwierig aufzutreiben war, in Uebersetzung der Mitwelt wieder zugänglich gernacbt. Die der Krankheit eigentümlichen Züge sind, wie bekannt, das Auftreten von choreatischen Bewegungen, welche nach Strümpell (20) mit denen der Chorea minor völlig übereinstimmen, und zwar selten vor dem 30 . Lebensjahre, meist erst im vorgerückten Alter, dabei zunehmende Geistesschwäche bis zur völligen Verblödung, und die Neigung, sich auf spätere Generationen zu vererben und meist zahlreiche Mitglieder einer Familie in mehreren aufeinanderfolgenden Geschlechtern zu befallen. Eine sehr ausführliche kritische Darstellung der Chorea Huntington verdanken wir Facklam (4) (1898).

In den letzten Jahren sind eine ganze Reihe von Publikationen über die Chorea Huntington erschienen, jedoch herrschen bezüglich einiger Punkte noch recht erhebliche Meinungsverschiedenheiten, so besonders über die Aetiologie und die pathologische Anatomie der Erkrankung, während man sich über die Symptomatologie, die Proguose 
usw. ziemlich einig ist. Ueber die Aetiologie der Erkrankung weiss man noch nicht viel; soviel steht fest, dass die meisten Fälle durch Vererbung erworben werden. Woher aber stammt die Erkrankung bei dem zuerst erkrankten Ahnen, der sie auf seine Nachkommenschaft überträgt? Es liegt auf der Hand, dass ihr noch andere Ursachen zu Grunde liegen müssen. Einzelne Fälle sind beschrieben worden, bei denen nichts von Heredität nachweisbar war. Man hat Traumen dafür verantwortlich gemacht und einen Zusammenhang mit Gemütserschütterungen, Schwangerschaft, Epilepsie, Migräne und dergl. angenommen [Westphal (23), Schabad (15)] u. a.

Auch bezüglich der patbologischen Anatomie der Krankheit sind die Verhältnisse durchaus noch nicht klar. Es erscheint daher zweckmässig, möglichst alle ärztlich beobachteten Fälle zu veröffentlichen, um Naterial für weitere Forschungen zu liefern. In den nachstehenden Zeilen möchte ich die Krankheitsgèschichten von drei Fällen von Chorea Huntington, die in den letzten Jahren in unserer Austalt (Direktor San.-Rat Dr. Lantzius-Beninga) beobachtet wurden, der Oeffentlichkeit übergeben, da mir einige Befunde nicht ohne allgemeines Iuteresse zu sein scheinell.

1. Sch., Johann, Landwirt aus K., ehelich geboren 1850. Eltern nicht blutsverwandt. Der Vater, seines Zeichens Schmied und Landwirt, körperlich und geistig gesund, wurde 62 Jahre alt und starb an einer "entzündeten Warze" an der Backe (wohl Infektion). Die Mutter worde $70 \mathrm{Jahre}$ alt. Von ihr aus ist der Patient erblich belastet. Sie war in den letzten Lebensjahren nervenund geisteskrank. Aus den Akten geht hervor, dass sie im hohen Alter an ${ }_{n}$ Nervenzucken" und Krämpfen gelitten hat. Der ärztliche Fragebogen besagt direkt: $n$ Mutter leidet an Veitstanz und ist unzurechnungsfähig " (die Mutter lebte damals, 1896, noch, der Fragebogen ist vom Hausarzt ausgefüllt). In einer Anstalt ist die Mutter offenbar nicht gewesen. Nach glaubwürdiger Aussage eines Ortseinwohners (Vormund des später unter 2. zu erwähnenden Patienten Theodor Sch.) haben noch 2 Geschwister der Mutter an derselben Krankheit (Nervenzucken) gelitten und sind daran gestorben. Nähere, Angaben darïber fehlen.

Ueber die Jugendjahre des Patienten sind die Angaben nur spärlich. Die körperliche und geistige Entwickelung verliefen anscheinend ohne Störungen. Patient war Soldat, später Bergmann; während dieser Beschättigung erwarb er eine ziemlich beträchtliche Schwerhörigkeit, anscheinend infolge einer Pulverexplosion. Lues wurde angeblich nicht akquiriert, jedoch scheint es, als ob er sich später dem Trunke ergeben habe. Er verheiratete sich. ${ }_{n}$ Ehestand und Trunk haben einen ungünstigen, nachteiligen Einfluss auf den Kranken ausgeübt" (ärztl. Fragebogen). Aus dem Anfang der 90 er Jahre wird berichtet, dass er in Unfrieden mit seiner Frau lebte, dieselbe misshandelte und bedrohte. Er beschuldigte seine Frau der ehelichen Untreue und behauptete sogar, dass 
sie mit ibrem eigenen Vater geschlechtlichen Verkehr habe, ausserdem treibe sie Unzucht mit dem Ortsnachtwächter. Die gereizte Stimmung des Patienten wurde durch übermässiges Trinken noch gesteigert, so dass er schliesslich gegen seine Familie aggresiv wurde und wegen Gemeingefährlicbkeit im Jahre 1893 der Irrenanstalt E. zugeführt werden musste.

Aus dem damals aufgenommenen Status ist folgendes bemerkenswert:

Patient sieht liörperlich kräftig aus, hat blühende Gesichtsfarbe. Infolge seiner Schwerbörigkeit ist eine Verständigung mit ihm mit Schwierigkeiten verknüpft. In seinem Verhalten ist er sehr stumpf und apathisch, ohne jede Krankheitseinsicht; sein gewalttätiges Verhalten seiner Frau und seinem Schwiegervater gegenüber, die er wiederholt mit Totschlagen bedroht hatte, leugnet er einfach $a b$. Die Sprache ist schwerfällig, stotternd, schwer zu verstehen. Sein Körper, besonders die Extremitäten, sind beständig in z a p pelnder, choreaartiger Bewegung, so dass sein Gang etwas Ungeschicktes bat. An seinen Wahnideen (Untrẹue der Frau) hält er fest, besteht darauf, sie zu verklagen und sich scheiden zu lassen. - Patient, dessen Erkrankung als Paranoia mit sekundärem Schwachsinn aufgefasst wurde, blieb 2 Jahre in der Anstalt, verhielt sich meist ruhig, half mit bei häuslichen Arbeiten. Er glaubte zuletzt, seine Frau sei gestorben, man habe es ihm zugerufen. Als seine Frau ihn besuchte, schien er nicht recht mit sich im Reinen, ob sie es wirklich wäre oder nur ein Phantasiegebilde; er äusserte wenigstens binterher, es könne auch jemand Anderes gewesen sein.

1895 wurde er entlassen. Bald nach seiner Entlassung geriet der Kranke mit seiner Umgebung wieder in Konflikt. Wenn er aufgeregt war, misshandelte er seine Frau tätlich und beleidigte sie in rohen und gemeinen Ausdrücken, vernachlässigte seine häuslichen und pekuniären Verhältnisse, zeigte anffallende Gedächtnisdefekte. Mitunter war er wieder, ,auffallend still ${ }^{4}$. In dem damals (1897) erstatteten Gutachten des Kreisphysikus heisst es: „Sprache stockend und schwerfällig. Patient macbt schon beim ersten Anblick den Eindruck eines schwachsinnigen Menschen"s. Der Gutachter hielt die Frkrankung für progressive Paralyse. Auf Grund dieses Gutachtens kam Sch. 1897 wieder in die Anstalt $\mathrm{E}$.

In den ärztlichen Akten wird angegeben:

Sch. zeigt dasselbo Verhalten wie bei seinem ersten Aufenthalt. Fr ist im allgemeinen stumpf und apathisch und sehr schwer zum Sprechen zu bewegen. Seine Intelligenz hat noch bedeutend mehr abgenommen, die Gedächtnisschwäche tritt noch auffallender hervor. Krankheitseinsicht fehlt völlig, er will entlassen sein, da ihm nichts fehle. Patient nimmt Teil an häuslichen Arbeiten.

1898 ist vermerkt: ${ }_{n}$ Patient ist stets stumpf und teilnahmslos, wird geistig immer schwäoher. Die Sprache ist so schwerfällig und unbebolfen, dass er kaum verständlioh ist". Er wurde immer hinfälliger, konnte fast nichts mehr arbeiten, klagte über Schmerzen in oberen und unteren Extremitäten, ging sebr steif- und breitbeinig. Geistig wurde er auch immer stumpfer und 
sprach fast gar nichts mehr, verkehrte mit Niemanden. Der Ernährungszustand war noch immer relativ gut.

1900 wurde der Patient in unsere Anstalt überführt. Die Untersuchung ergab, wie früher, das Vorhandensein charakteristischer choreatischer Zuckungen in oberen and unteren Extremitäten und Rumpfmuskulatur. Dio Haltung ist steif, der Kopf etwas nach hinten und links gezogen; der Gang ungelenk, schiebend, meist hilft Sch. beim Gehen in der Weise nacb, dass or den ganzen steifgehaltenen Körper um die Iaängsachse dreht. Die Zungo macht unsichere Bewegungen, weicht etwas nach links ab und wird beim Sprechen fast gar nicht bewegt, so dass die Antworten nur dann zu verstehen sind, wenn man schon vorher ungefähr weiss, was er sagen will. Im übrigen ist Sch. ganz verblödet, ausser seinem Namen weiss er kaum noch etwas anzugeben, beschäftigt sich nicht, verkehrt nicht. Aussehen ungesund, Gesichtsfarbe blass und zyanotisch, Herzaktion schlecht. Patient bewegt sich wenig, sitzt meist oder liegt.

Der' Tod erfolgte 1901, der Kranke erlag einer hypostatischen Pneumonie infolge chronischer Herzschwäche (kleines, atrophisches Herz).

Das sehr ansführliche und genaue Sektionsprotokoll verzeichnet wörtlich :

„Das Gehirn mit seinen Häuten bietet in allen Teilen normale Verbältnisse; insbesondere sind Stirn- und Schläfenwindungen äusserlich und im Durchschnitt normal, Schnitte durch die Stammganglien, Pons, Medulla oblongata und Kleinhirn ohne Besonderheiten. Pia zart, ebenso alle Gefässe. Nur die rechte Vertebralis ist bedeutend kleiner als die linke. Blutgehalt normal."

2. Sch., Theodor, Bruder des vorigen, Gast- und Landwirt, 1854 geboren.

Nach den Angaben des ärztlichen Fragebogens war Patient als Kind gesund, hatte nie Krämpfe, war aber von jeher leicht zornig und reizbar. Keine syphilitiscbo Ansteckung, keine sonstigen nachteiligen Einflüsse der Verhältnisse auf seine Gesundheit. War Soldat, verheiratete sich, hatte 4 Kinder. Die Krankbeit kam bei ihm zum Ausbruch otwa 1904, also ungefähr im Alter von 50 Jahren. Sie begann allmählich mit Vorläufern (Hör- und Sprachstörungen), bald stellte sich auch das ominöse „Nervenzucken" ein. Die Sprache verschlechterte sich immer mehr, sie wurde stammelnd, der Gang unsicher. Nach etwa 2jähriger Dauer des Ireidens (1906) musste Patient sein Geschäft aufgeben, da er nicht mehr im Stande war, ein Glas Bier oinzuschänken. Er verpachtete seine Wirtschaft und wohnte bei dem neuen Inhaber derselben. Er wurde aber immer hinfälliger, unsauber mit seinen Entleerungen; zuletzt zog er sich nicht mehr aus und duldete auch nicht, dass andere ihn entkleideten. Dadurch, dass er seine Notdurft auf der Strasse verrichtete, erregte er öffentliches Aergernis und wurde nun auf Grund oines kreisärztlichen Attestes in unsere Anstalt aufgenommen (1909). Bei seiner Aufnahme war er völlig teilnahmslos und ohne Verständnis für seine Lage. Körperlich war er sehr schmutzig und verwahrlost; gegen Auskleiden und Baden sträubte er sich in 
der heftigsten Weise, wollte nicht im Bett bleiben, wurde erst auf Narkotika ruhiger. Am Tage nach der Aufnahme ganz verwirrt und verblödet, nicht zu fixieren. Lebhafte choreatische Bewegungen mit Kopf, Händen, Armen, lebhaftes Grimassieren. Spricht wenig und nur in abgebrochenen Sätzen, schreit laut, wenn er ins Bad soll.

Status praesens: Mittelgrosser, kräftig gebauter Mann. Knochenbau und Muskulatur gut entwickelt. Reflexe nicht wesentlich verändert, nurPatellarreflex etwas gesteigert. Fussklonus angedeutet, kein Babinski. Sensibilität anscheinend herabgesetzt, ihre Prüfung mit Schwierigkeiten verbunden. Motilität: Patient geht unter öfteren Drehungen in der Hüfte und Einknicken in den Kniegelenken. Mit Gesicht und Kopf macht er fortwährend ungeordnete, choreaartige Bewegungen, dreht den Kopf rechts und links, beugt ihn etwas vor und wieder zurück in schraubenförmigen Windungen, faltet die Stirn, macht die Augen auf und zu, bildet den Mund zur Schnauze und verzerrt ihn bald so, bald so, dabei halblaute Töne van sich gebend. Auch die Hände sind in fortwährender Bewegung; die Muskulatur der Hände zittert. Starker Spasmus besonders in Knie- und Hüftgelenken und im Nacken. Sprache unverändert infolge der choreatischen Bewegungen der Lippen, der Zunge, des Gaumens, halblautes kindliches Lallen, Zunge liegt wie ein Kloss im Munde. Gesichtsausdruck ganz verblödet. Pupillen mittelweit, rund, gleich, prompt reagierend. Auf alle ihm vorgelegten Fragen reagiert Patient damit, dass er unter lebhaftestem Grimassieren kaum verständlich seiner Namen nennt.

Der Zustand ist bis heute im wesentlichen unverändert geblieben. Die choreatischen Bewegungen haben an Intensität nachgelassen, nehmen aber bei kirregung wieder zu. Im Schlaf sind sie nicht bemerkbar. Patient ist sebr unsauber mit seinen Entleerungen; ein beginnender Dekubitus ist unter Bäderbehandlung wieder abgebeilt. Sein geistiger Zustand muss als totalo Verblödung bezeichnet werden.

3. B., Gustav, Schreiner. 1856 ehelich geboren. Von den Eltern ist nur bekannt, dass sie früh starben; nähere Angaben über Kindheit fehlen. Patient war ein fleissiger und ordentlicher Arbeiter, trank nicht; ist verheiratet. 1895 erlitt er einen Unfall, indem ihm ein schwerer Balken auf den Kopf fiel; er war damals einige Zeit bewusstlos, hatte eine Wunde am Kopf. Erbrechen worde nicht beobachtet. Er lag wochenlang zu Bett; nach $\Lambda$ ngabe seiner Frau und Tochter soll damals Sprachstörung bestanden haben. Seit dem Unfall hatte er fast anhaltend $K$ opfschmerzen, auch liess das Gedächtnis nach, er war aber noch im Stande, weiter zu arbeiten. Auch wollen die $A \mathrm{n}$ gehörigen bald nach dem Unfall das Auftreten von $n^{\text {zuckenden Bewegungen }}$ mit dem Kopf", die allmählich an Intensität zunahmen, beobachtet haben. Seit 1901 fiel es auf, dass der Gang unsicher wurde. Anfang 1903 überstand er eine fieberhafte Erkrankung (lnfluenza) mit rasenden Kopfschmerzen. Im Anschluss daran zeigten sich Symptome nächtlicher Unruhe, er schlug mit den Händen auf die Bettdecke, kratzte mit den Nägeln an derselben, stöhnte laut, wiederholte oft dieselben Worte, wobei man bemerkte, dass die Sprache un- 
sicher und schwer verständlich war. Patient war dabei noch orientiert, lannte seine Umgebung, war aber, in Gegensatz zu seinem bisherigen Verhalten, auf einmal ausserordentlich reizbar (ein Lattengeländer, an dem er aus Versehen bängen geblieben war, wollte er zertrümmern, auch wollte er: einen Tisch zerschlagen, weil die bestellten Kohlen nicht pünktlich eingetroffen waren). Auf Anraten des Arztes wurde er am 5.3.03 in die städtische Irrenanstalt in F. gebracht. Das Vorliegen einer erblichen Veranlagung wurde von den Angehörigen entschieden in $A$ brede gestellt.

Aus dem damals aufgenommenen Status entnehme ich Folgendes:

nPatient kann nicht ruhig stehen, er befindet sich fast fortwäbrend in Unruhe, indem deutliche choreatische Bewegungen an Armen, Beinen, Rumpf, Kopf und im Gesicht anftreten, die durch willkürliche Bewegungen an sich gemindert werden. Sobald man den Patienten anredet, nehmen die ungewollten Bewegungen deutlich au Intensität zu; sehr stark sind sie auch, wenn Patient etwas erzählen will. Soll der Kranke, auf einem Beine stehend, Häude und Zunge vorstrecken, so wird die Unrube so lebhaft, dass er das Gleichgewicht verliert. Pupillen gleich, mittelweit, reagieren nur träge. Sprache undeutlich, verwaschen. Zunge wird gerade vorgestreckt, von groben Zuckungen bewegt. Patellarreflexe sehr gesteigert, aber kein Patellarklonus. $\Lambda$ chillessehnenreflex lebhaft, Andeutung ron Fussklonus. Keine Spasmen oder Paresen in den unteren Extremitäten. Gang leicht tänzelnd, aber sicher. Kein Romberg. An den oberen Extremitäten sind die Sehnenreflexe nicht merklich gesteigert, keine Spasmen oder Paresen, kein Tremor der Finger.

Patient berichtet, dio Chorea sei bei ilm vor 2 Jahren, 1901, aufgetreten. Erst habe er es in den Beinen verspürt, dann in Armen und Gesicht. Auch habe er damals im Kopf eine Art Schwindel gehabt. Die Bewegungen seien nie so stark gewesen, dass sie ihn am Essen oder Sprechen hinderten. Zur Zeit hält sich Patient für gesund, abgesehen vom „Zittern", hat keine Einsicht, bestreitet seine grosse Reizbarkeit, will auch Gedächtnisschwäche zuerst nicht zugeben; nach einigen Proben im Kopfrechnen gibt er aber zu, früher sei das Gedächtnis besser gewesen. Er ist orientiert und geordnet. ${ }^{*}$

Nach einigen Wochen Aufenthaltes in der Anstalt wurde er als gebessert entlassen, aber bereits im Mai 1903 machte sich die $\mathrm{Ne}$ u a u $\mathrm{n}$ a b me notwendig. Er hatte in der $Z$ wischenzeit vergeblich versucht, die Arbeit wieder aufzunehmen, war sebr gereizt, aufgeregt, die Zuckungen wurden wieder stärker, er verschüttete das Essen, schinipfte, wenn er ermahnt wurde, war dabei sehr vergesslich, fand eines Tages eine Wohnung nicht, in der er am Tage zuvor noch gewesen war, klagte über Kopfschmerzen.

Bei der Aufnahme erwiesen sich die choreatischen Zuckungen, die bei der Entlassung ziemlich gering gowosen waren, als wieder sebr stark vorhanden. Wegen der lobhaften motorischen Unruhe konnte er die Speisen nicht zum Munde führen; beim Anreden trat eine deutliche Steigerung der choreatischen Bewegungen ein. Allmählich besserte sich auch diesmal der Zustand. Wenn er sich unbeobachtet fühlte, z. B. beim Lesen der Zeitung, konnte der Kranke 
ganz ruhig dasitzen. Im Juli 03 wurde er von seinen Angehörigen wieder abgeholt.

Nun ging es einige Jahre ganz gut mit ihm, es war eine Art Romission des Leidens eingetreten. Er war im Stande, seiner Arbeit in den Eisenbahnwerkstätten wieder nachzugehen, war auch $p$ sychisch nicht besonders auffallend, blos ein bischen eigen, gleich aufgeregt, tat gern das Gegenteil von dem, was man ihm sagte, war aber immer gutmütig. Die Zuckungen waren fast völlig verschwunden, nur die Sprache war nach wie vor undeutlich. Eine neue Verschlimmerung trat erst 1906 ein und zwar wiederum im Anschluss an ein K o p f trauma (Patient war von einem Kollegen vor die Brust gestossen worden, stürzte rückwärts nieder und scblug mit dem Kopf an die Bank an). Er war zwar nocb im Stande, nach Hlause zu gehen, musste aber am anderen Tage bereits liegen bleiben, hatte einige Male Erbrechen und so trat nun eine rasch zunehmende Verschlechterung seines Befindens ein. Vor allem wurde die Sprache sehr undeutlich, zeitweise unverständlich. Dic Zuckungen wurden äusserst heftig und besonders beim Gehen so stark, dass er einige Malo auf der Strasse stürzte. Auch psychische Erscheinungen traten stärker in den Vordergrund, der Kranke war wieder erregt, drängte immer fort, war nicht jm Hause zu halten, wollte bald dies, bald das. An 2 . Juni 06 musste er wiederum in die Irrenanstalt (3. Aufnabme).

Status: Muskelzuckungen bald hier bald dort in der gesamten Körpermuskulatur, ganz unregelmässig, auch völlig unkoordiniert; manchmal kontrabiert sich nur ein einzelner Muskel, manchmal mehrere gleichzeitig, aber nicht im Sinne einer zweckmässigen Bewegung. Die Intensität der Muskelkontraktionen wechselt ebenfalls sehr stark. Die beiden Körperhälften sind in gleicher Weise beteiligt, am Rumpf besonders die Strecker und Dreher der Wirbelsäule, aber auch in der Bauchmuskulatur treten öfter Kontraktionen auf. Die Gesichtsmuskulatur, ebenso die Zunge ist beteiligt, auch anscheinend Gaumen- und Kehlkopfmuskeln, so dass die Sprache ruckweise, schnalzend, manchmal ganz unverständlich wird. Der Gang ist ebenfalls durch die sich eirschiebenden unkoordinierten Bewegungen gestört, doch kann Patient, wenn man ihn direkt dazu auffordert, für einige Zeit die Bewegungen unterdrücken und es gelingt ihm, Sprache und Gang fast normal zu gestalten. - Patient ist orientiert, hat Krankheitsgefübl, gibt zu, dass er zu Hause aufgeregt sei, dass er eine Kur durchmachen wolle, dass ihm der Aufenthalt in der Anstalt sohon einmal gut getan habe. - Obne dass eine wesentliche Besserung eingetreten wäre, wurdo der Kranke am 10. 8.06 nach Hause abgeholt, am 17. 8. aber wieder aufgenommen (4. Aufnahme). Er war zu Hause wieder sehr unruhig, lief den ganzen Tag umher, bald im Keller, bald auf der Mansarde, war gereizt und zornig, schimpfte viel, blieb nachts nicht im Bett, hatte wieder sehr heftige Zuckungen. Mit einer ganz kurzen Unterbrechung von 3 Tagen ist der Kranke seit dieser Zeit in der Anstalt. Der Zustand wie früher; für kurze Zeit kann Willensanstrengung die unwillkürlichen Bewegungen etwas hemmen. Sprache völlig unverständlich, Schlucken mühsam. - Seit November 08 befindot sich der Kranke in unserer Anstalt. Er ist ruhig, freundlich, spricht nicht viel. 
Infolge der bei jeder Bewegung auftretenden heftigen schleudernden Bewe gungen von Kopf und Extremitäten ist er sehr hilflos und liegt meist \%u Bett. Er kann noch allein gehen, jedoch bat sein Gang infolge der dabei auftretenden Drehungen und Windungen des Körpers und der Schleuderbewegungen der Beine etwas sehr Grotesles. Der 'Zuschauer ist jedesmal froh, wenn Patient wieder glücklich im Bett gelandet ist. Mit dem Essen wird er, trotz der Bewegungsstörung, ganz gut fertig; er weiss mit grossem Geschick den Löffel zu dirigieren und so rasch zum Munde zu führen, dass gar keine Zeit bleibt zum Verschütten. Seine Sprache ist kaum mehr verständlich. Psychisch ist er bereits recht schwachsinnig, er verrät wenig Interesse für die Vorgänge in seiner Umgebung, ist teilnahmslos, dabei meist barmlos freundlich. In der Ruhe lassen die ungewollten Bewegungen sichtlich nach, wenn sie auch nicht völlig scbwinden. Auch im Schlaf schwinden sie nicht ganz.

Wenn ich die wesentlichsten Erscheinungen der drei Fälle noch einmal kurz zusammenfasse, so sind es folgende:

Bei Fall 1: Erbliche Belastung von der Hutter. Schädliche Einflüsse: Alkohol. Auftreten der Erkrankung im Anfang des fünften Lebensjahrzehntes (etwa mit 43 Jahren). Die Psychose zeigt anfänglich stark paranoische Zügre (Alkoholparanoia?), die aber später ganz zurücktraten. Der progressive Charakter zeigt sich in rasch zunelimender Demenz. Dauer des Leidens 9-10 Jahre. Sektionsbefund bezüglich des Gehirns makroskopisch negatir, mikroskopische Lntersuchung leider unterblieben.

Bei Fall II: Erbliche Belastung wie bei I. Von schädlichen Einflüssen Potus wahrscheinlich, keine Lues. Auftreten der Erkrankung am Ende des fünften Lebensjahrzehntes (ó. Lebensjahr). Rasche Ausbildung einer hochgradigen Intelligenzstörung bei verbältnismässig wenig intensiven choreatischen Zuckungen. Dauer des Leidens bis jetzt 5 Jahre.

Bei Fall 1II: Anscheinend keine direkte Vererbung, vielleicht aber allgemeine neuropathische Belastung ${ }^{1}$ ). Kein Potus, keine Lues. Auf-

1) Es ist hierzu Folgendes zu bemerken: Während, wie oben erwähnt, bei der ersten Aufnahme des Kranken in die Anstalt die Angehörigen das Vorliegen einer erblichen Veranlagung entschieden in $\Lambda$ brede stellten, hat die Frau jetzt, bei Abschluss dieser Arbeit, bei einer nochmaligen Anfrage angegeben, die Mutter des Patienten sei in einer Anstalt gestorben, so viel sie gehört habe, an "Tiefsinn". Eine Schwester des Patienten sei vor einigen Jahren an demselben Jueiden gestorben, wie es jetzt ihr Mann habe (?). Eine andere Schwester sei vor längeren Jahren $2 \mathrm{mal}$ in einer Anstalt gewesen, habe sich abor vollständig wieder exholt. - Welcher Wert bei den offenkundigen Widerspr üchen den Bekundungen der Frau beizumessen ist, muss ich dahingestellt sein lassen. Es muss aber nach dem Mitgeteilten auch in diesem Fall mit der Möglichkeit, wenn nicht einer direkten Vererbung, so doch einer neuropathischen Veranlagung gerechnet werden. Ieider haben sich diesbezüglich keine genaueren Feststellungen machen lassen. 
treten der Frkrankung mit etwa 40 Jahren, und zwar stellten sich die Anfangssymptome kurze Zeit nach einem Unfall (Kopftrauma) eill. Vorübergehende Remission, und neue Verschlimmerung offenbar wieder in direktem Anschluss an ein neues Trauma (Fall auf den Kopf). Demenz mässigen Grades, dafür hochgradige Intensität der choreatischen Erscheinungen. Dauer des Leidens bis jetzt 12 Jahre.

Es ergibt sich nun aus den vorliegenden Beobachtungen, dass zunächst in ätiologischer Hinsicht zwei von meinen Fällen eine ausgesprochene choreatische Heredität zeigen, beido Kranke sind Mitglieder einer Choreafamilie. Bei dem dritten Falle konnte eine hereditäre Belastung nicht mit Sicherheit festgestellt werden; es scheint in der Tat eine solche nicht zu bestehen, da sie von den Angehörigen bestimmt bestritten wurde, jedoch scheint eine neuropathische Veranlagung der Familie vorhanden zu sein.

Wenn ich einige der in den Zeitschriften in den letzten Jahren veröffentlichten Fälle zum Vergleich heranziehe, so ersieht mav, dass über die Aetiologie der Erkrankung auch heute noch die Meinungen auseinandergehen. Soviel steht fest, dass die meisten Fälle durch Vererbung erworben werden. Hierzu macht Antoni d'Ormea(13), der 1904 eine Choreafamilie aus Ferrara beschrieb, von der bis dahin vier Generationen an Chorea Huntington litten, während die fünfte allerdings noch in jugendlichem Alter stehende, noch frei geblieben war, einen gewiss beherzigenswerten Vorschlag. Mit Rücksicht auf den Limstand, dass in der 4. Generation nur ein männliches Individuum trotz seiner 49 Jahre verschont blieb, und dass gerade dieser Mann von seiner Mutter, die bei seiner Geburt starb, nicht genährt wurde, fordert er, man solle, wemn man nicht überbaupt die Ehe solcher Kranken inhibieren könnte, was schliesslich das beste Vorbeugungsmittel sej, wenigstens das Stillen von seiten einer kranken Mutter energisch verbieten und dem. Neugeborenen die Nahrung von einer absolut gesunden Frau verschaffen.

Eine andere Choreafamilie ist von Curschmann(2) geschildert worden. In dieser Familie war ausgesprochene Demenz überhaupt nicht regelmässig, sondern sogar ziemlich selten aufgetreten. Es liess sich zwischen der Demenz und der Stärke der choreatischen Erscheinungen, welche letzteren sehr erheblich waren, eine deutliche Inkongruenz konstatieren. Nach der Ansicht Curschmanns spricht dieser Umstand gegen die von einigen Autoren geltend gemachte Annahme, dass die psychischen Störungen einfach als direkte Folgeerseheinungen der motorischen aufzufassen sind, beide sind vielmehr koordinierte Reizbezw. Ausfallserscheiuungen auf Grund desselben Prozesses, dessen 
Ein Beitrag zum Krankheitsbild der Chorea chronica progressiva. 799

Sitz für die psychischen Störungen wahrscheinlich mehr im Stirnhirn, für die motorischen in den Bewegungszentren der Rinde zu suchen ist. - Die erwähnte Inkongruenz zwischen Demenz und Stärke der choreatischen Zuckungen zeigen übrigens unsere Fälle II und III ebenfalls.

Weyrauch (22) hat zwei Fälle von Chorea Huntington veröffentlicht, die einer Choreafamilie entstammeu, deren Stammvater an Chorea litt und ein sehr ausschweifendes Leben geführt haben soll. In drei aufeinanderfolgenden Generationen kamen acht charakteristische Fälle zur Beobachtung. In säntlichen Beobachtungen konnte beträchtliche Geistesschwäche nachgewiesen werden. Bei 6 Mitgliedern der Familie trat das Leiden zwischen dem 30. und 43. Lebensjahr auf. Nach demselben Autor springt die Erkrankung nie vom 1. bis in das 3. Geschlecht. Hierzu vergleiche man den uoch später zu erwäbnenden Fall von Engelen(3), in dem die Vererbung durch ein selbst verschont gebliebenes Familienmitglied erfolgte.

Der von Nerlich (12) veröffentlicbte Fall bietet nichts Besonderes. Der Patient stammte aus einer belasteten Familie; mit 32 Jahren traten choreatische Zuckungen auf, die sich im Laufe der Jahre verschlimmerten und zu denen schliesslich völlige Verblödung hinzutrat.

Ein von Bonfigli(1) mitgeteilter Fall weicht in manchen Punkten von der Regel ab. Erbliche Anlage fehlte, doch war Tuberkulose in der sehr gliederarmen Familie. Der Beginn zeichnete sich durch starke Steigerung der Libido aus. Der Verlauf war kürzer als gewöhnlich (7 Jahre). Die Sprachbehinderung trat erst spät ein; die Reflexe waren abgeschwächt, statt wie sonst gesteigert.

$\mathrm{Zu}$ den Fällen, bei denen eine hereditäre Belastung nicht nachweisbar war (wie anscheinend bei meinem Fall III), gehört der von Frank (3) beschriebene. Derselbe betraf eine 43jährige Frau, bei der bestimmt keine Heredităt vorlag. Die Patientin erkrankte plötzlich, über Nacht, an choreatischen Zuckungen im rechten Arm, die allmählich auf den übrigen Körper und das Gesicht übergriffen. Die Bewegungen börten in der Nacht oft nicht auf, ferner trat häufig nächtliches Zungenschualzen und Zähneknirschen auf. Abweichend vom typischen Krankheitsbild waren ferner die Verminderung der Sehkraft und die Abschwächung des Gehörsinnes. Die Reflexe waren normal, nicht gesteigert. Psychische Symptome waren Reizbarkeit und Störungen der Intelligenz von progressivem Charakter. Jede Therapie erwies sich als nutzlos. In dem vollständigen Fehlen des hereditáren Momentes erblickte Verfasser mit Recht eine auffallende Erscheinung.

1902 hat Westphal (23) zwei Fälle von Chorea Huntington mitgeteilt. Der erste war hereditär vom Vater belastet, der gleichfalls an 
Chorea Huntington gestorben war. Die Patientin erkrankte nach der Geburt des jüngsten Kindes im Wochenbett an Chorea. Die Zuckungen nahmen mit den Jahren an Intensität zu und betrafen schliesslich die gesamte willkürliche Muskulatur. Beim Sprecben gab die Patientin eigentümlich schnalzende Laute von sich. Komplizierte Bewegungen, wie An- und Ausziehen, waren nur sehr mühsam und unter grossem Zeitverlust ausfübrbar. Die Kniephänomene waren gesteigert, sonst körperlich nichts Abnormes. Dabei bestand grosse Reizbarkeit, im Anfang Wutanfälle, später Nachlass dieser Affektstörungen mit zunehmender intellektueller Schwäche.

Bemerkenswert an diesen Fall war besonders, dass das Wochenbet t bei bestehender erblicher Veranlagung die Gelegenheitsursache für das Auftreten der Cborea abgab. - Der zweite Fall hat sich nicht auf dem Boden der Heredität, weder der gleichartigen Vererbung, noch der neuropathischen Belastung entwickelt, sondern ist im Anschluss an ein schweres Trauma entstanden (Sturz vom Gerüst, 3 Stock hoch, Verletzungen Kopf und Kreuzbein). Bald nach der Entlassung aus dem Krankenhause haben sich dann die ersten choreatischen Bewegungen eingestellt und sich zu der jetzt bestehenden schweren Bewegungsstörung entwickelt. Sämtliche Muskelgruppen nahmen Teil an der choreatischen Unrube, die Sprache war ein kaum verständliches Lallen. Bei gewollten Bewegungen liessen die choreatischen Bewegungen sichtlich nach, auch beim ruhigen Liegen im Bett erfolgte deutliches Nachlassen. Kniephänomene waren lebhaft gesteigert, kein Fussklonus. Psychisch bot sich das Bild des apathischen Blödsinns.

Der Fall hat einige Aehnlichkeit mit meinem Fall III, der sich ja auch in direktem Anschluss an eine heftige Gewalteinwirkung auf den Kopf entwickelt hat. Westphal meint, dass wir in derartigen sporadischen Fällen vielleicht die Stammväter einer choreatischen Deszendenz vermuten dürfen.

Vou anderen veranlassenden Momenten für das Zustandekommen des Krankheitsbildes werden vereinzelt auch heftige Gem ütserschütterungen u. dergl. angegeben. Skoczyuski(17) stellte in der Gesellschaft der Berliner Charitéärzte 1902 eine 68 jährige Frau vor, bei der sich im Anschluss an einen heftigen Schrecken während eines grossen Feuerschadens im Hause der Patientin eine progressive Chorea langsam entwickelte. Die Frau war nicht hereditär belastet.

Ein Fall von chronischer Chorea, bei dem ebenfalls eine erbliche Belastung nicht aufzufinden war, und der sich an eine langjährige Migräne anschloss, ist von Schabad (15) mitgeteilt worden. Es handelt sich um eine Frau, die, seit dem 22. Lebensjahre an Migräne 
Ein Beitrag zum Krankheitsbild der Chorea chronica progressiva. 801

leidend, mit 40 Jahren an Zuckungen im ganzen Körper erkrankt. Kopf und mimische lluskulatur sind in fortdauernder Luruhe; Schleuderbewegungen der oberen Extremitäten, fortwährende Beuge- und Streckbewegungen der Beine. Steigerung der Erscheinungen bei Aufregungen, Höglichkeit der Unterdrückung bei Willensanspannung, nahezu völliges Aufhören im Schlaf. Sprache ebenfalls deutlich gestört. Patientin ist noch nicht dement, doch ist "ibr Ideenkreis beschränkter wie früher". Auch dieser Fall dürfte der Chorea Huntington zuzurechnen sein.

Einen auffallend frühen Beginn des Leidens, nämlich bereits mit dem 20. Lebensjahre, zeigt der von Hoffmann (8) im Naturhist. med. Verein in Heidelberg demonstrierte Fall, der eine jetzt 34 jährige Frau betrifft mit sehr starker motorischer Luruhe bei weit vorgeschrittener Demenz.

Noch früher zeigte sich der Beginn der Krankheit in dem von Friedenthal (6) beschriebenen Fall. Die Erkrankung trat bereits im 12. Lebensjahr auf; sie zeigte alusserdem exquisit hereditären, degenerativen Charakter, fortschreitende Verblödung usw.

Im Verein der Aerzte Düsseldorfs stellt Engelen (3) 1906 einen Fall von Chorea Huntington vor, bei dem die Erkrankung mit Unsicherbeit der Hände begann, dann folgten die Beine, schliesslich Sprachstörung. Bei Willensanspannung war die Aussprache ziemlich deutlich, auch liessen sich die Zuckungen dann unterdrücken, sodass der Patient damn imstande war, zu essen, zu schreiben, die Treppo hinaufzusteigen. Ausser Steigerung der Sehnenrefexe war kein neurologischer Befund nachweisbar. Dieser Fall ist noch durch den Umstand besonders interessant, dass hier die Vererbung durch ein selbst verschont gebliebenes Familienmitglied erfolgte. Die Grossmutter des Patienten litt an dem Uebel, desgleichen ein Bruder des Vaters, der Vater selber dagegen nicht; zwei seiner Kinder aber bekamen die Krankheit.

Zwei an Chorea progressiva leidende Patienten sind 1907 von Wallenberg (21) im Aerztl. Verein Danzig besprochen worden. Bei dem ersten Fall trat die Erkrankung auch bereits auffallend früh, Mitte der $20 \mathrm{er}$ Jahre auf und war in ihrem Endstadium durch Gehstörungen, ataktische Bewegungen, taumelnden zerebellaren Gang, verwaschene bulbăre Sprache, Gedächtnisstörungen und Pupilleustarre charakterisiert; der zweite endigte mit einem an Dementia praecox grenzenden kindischen Wesen, Zwangshandeln usw., der erste Fall ein spiuales, der $z$ weite ein zerobrales Schlussbild derselben Krankheit.

Anknüpfend an einen Fall von Chorea chronica progressiva, bei dem der Beginn der Erkrankung ebenfalls in eine sehr frühe Lebens- 
periode, in die ersten Jahre des 3. Dezenniums fiel (es handelte sich um das erste erkrankte Glied der 4. Generation einer Choreafamilie) hat Heilbronner ( $\bar{l}$ ) die Frage aufgeworfen, ob etwa das Tieferrücken des Anfangsalters der Erkrankung in jeder folgenden Generation eine regelmässig zu beobachtende Erscheinung sei. Er neigt der Auffassung zu, dass der familiären Chorea im allgemeinen die Tendenz innewobnt, in jeder folgenden Generation im Durchschnitt jüngere Individuen zu befallen als in der vorhergegangenen und meint, in dieser Tendenz, in dem nicht nur in Bezug auf das Individuum, sondern auch auf den Stamm progressiven Verlauf "w ürde sich die degenerative Natur der Chorea Huntington besonders instruktiv dokumentieren".

Auch Curschmann (2) schliesst aus dem Stammbaum der von ihm geschilderten Choreafamilie, dass der Chorea Huntington die Tendenz innewohnt, von Generation zu Generation jnmer jüugere Individuen $z u$ befallen. "Nicht nur die Krankheit des Individuums ist eine progressive, sondern auch die familiäre Neigung zu ilhr".

$\mathrm{Ob}$ sich dies wirklich so verhält, müssen weitere Beobachtungen lehren. In meinen oben mitgeteilten Fällen sind die Kinder der beiden ersten Patienten bis jetzt gesund, der dritte ist kinderios. $\mathrm{Zu}$ bemerken ist hierzu, dass die Mlutter der beiden unter I and II beschriebenen Patienten (sie wurde 79 Jahre alt und war nach den Berichten erst in den "letzten Jahren" leidend) ihre Chorea offeubar erst spät bekommen hat, während ihre Söhne bereits mit 43 bezw. 50 Jahren erkrankt sind.

Ueber dio der Erkrankung zugrunde liegenden pathologischen Veränderungen herrscht noch Unsicherbeit. Oppenheim sagt darüber: „In vielen Fällen werden Veränderungen an den Ileningen, Trübung, Hydrocephalus externus, Pachymeningitis haemorrhagica gefunden. In diesen darf jedoch die Grundlage des Leidens nicht gesucht werden, da sie wohl immer akzidenteller Natur sind. In 2 Fällen fand ich dissemenierte, miliare enzephalitische Herde, besonders zahlreich in der Rinde und im Mark der motorischen Region (14a). Aehuliche Befunde wurden von Facklam, Kréaval-Raviot u. a. erhoben, doch lag in anderen Fällen eine diffuse lleningoencephalitis chronica mit sekundärer Atrophie der Hirnrinde vor. Binswanger erwähnt, dass sich in einem von ihm beobachteten Falle der Hirnbefund nicht wesentlich von dem der Dementia paralytica unterschicden habe. Lannois und Paviot sehen auf Grund ihrer Untersuchungen in der Wucherung der Neuroglia, in der Infiltration der Rinde mit Gliazellen das Wesentliche und wollen diesem Homent in der Pathogenese der here- 
Ein Beitrag zum Krankheitsbild der Chorea chronica progressiva. 803

ditär-familiären Nervenkrankheiten überhaupt eine bedeutende Rolle zuschreiben. Ob die angeführten Befunde das anatomische Substrat der hereditären Chorea bilden, steht noch dahin".

Der oben bereits erwähnte negative Sektionsbefund unseres Falles I dürfte mit obigem Ausspruche Oppenheims in Einklang stehen, dass in den groben Veränderungen an den Meningen usw. die Grundlage des Leidens nicht gesucht werden darf. Auch der von $\mathrm{Heyer}$ (11) 1907 im Aerztlichen Verein Danzig mitgeteilte Befund, nämlich das Vorhandensein einer dicken Schwarte in ganzer Ausdehnung zwischen Dura und Pia, ist wohl nur als Nebenbefund aufufassen. Alle neueren Veröffentlichungen verlegen die krankhaften Veränderungen in die Hirn. rinde. 1902 hat Stier (19) in der Med. nat. Gesellschaft in Jena über die pathologische Anatomie der Huntingtonschen Chorea gesprochen und zwar im Anschluss an einen 21/2 Jahre beobachteten und zur Sektion gekommenen Fall. Makroskopisch war nur ein starker Schwund des Gehirns zu finden, das Gewicht betrug nur $1073 \mathrm{~g}$, ausserdem eine leichte Erweiterung der Ventrikel und Verdickung des Ependyms; an den Häuten und am Rückenmark keine Veränderungen.

Mikroskopisch: Im Rückenmark keine nennenswerten Störungen ausser leichter Degeneration in einigen hinteren Wurzeln und isolierter Degeneration einzelner Nervenfasern in den verschiedensten Strängen. Im Gehirn zeigte das Marklager in allen Gegenden und Schichten eine gleichmässig verteilte, nirgends zu Herden verdichtete Vermehrung der Gliazellen; die grossen Stammganglien zugleich ein engeres $\mathrm{Zu}$ sammenliegen der Ganglienzellen und an einigen Stellen kolloide Entartung. Die Gefässe waren in der Rinde an Zahl vermehrt, ihre Struktur nirgends krankhaft verändert. Die Hirnrinde fast überall verscbmälert, überall Schwund der Tangentialfasern, deutliche Vermehrung der kleinen runden Zellen und Verminderung und ungeordnete Lagerung der Ganglienzellen. Die letzteren waren nur zum kleinen Teil normal, die meisten wiesen alle Zeichen der beginnenden und fortgeschrittenen chronischen Erkrankung auf. Am stärksten waren die Destruktion der Ganglienzellen und die Wucherung der Glia ausgeprägt in den motorischen Zentren, am geringsten in der Stirnrinde.

Weyrauch (22) meint, dass man bei der progressiven Chorea neben anderen organischen Veränderungen gewöhnlich Atrophie der Hirnrinde findet; sie ist als chronisch disseminierte oder diffuse Enzephalitis anzusprechen. Auch Lebram (10) hat darauf hingewiesen, dass nach den im Königsberger Pathol. Institut gemachten Untersuchungen chronisch entzündliche Prozesse (Encephalitis chronica) eine Rolle spielen. 
Bonfigli (1) fand in einem Falle Veränderungen, die den paralytischen Gehirnbefunden analog waren, der Unterschied bestand nur in dem Fehlen von Plasmazellen.

1908 hat Schula (16) in einem Falle von Chorea progr. Verwachsung der Dura mit dem Schädeldach und Leptomeningitis geringen Grades, leichten Hydrocephalus externus, geringe Atrophie des gesamten Gehirnes gefunden, daneben aber noch ausgedehnte Veränderungen im Inneren der Ganglienzellen, besonders der grossen, zum Teil auch der kleinen Pyramidenzellen; vereinzelte Veränderungen der Ganglienzellen im Rückenmark usw. Auch er ist der Ansicht, dass der eigentliche primäre Krankheitsprozess sich in der Hirnrinde abspielt und in degenerativen Veränderungen der Ganglienzellen der Hirnrinde besteht. ${ }^{1}$ )

Noch einige Worte über die Behandlung der Chorea Huntington. Dieselbe ist recht aussichtslos. Ilan muss nach Köster (9) versuchen, die Zuckungen durch die bei der Chorea minor erprobten Beruhigungsmittel günstig zu beeinflussen. Mehr oder weniger haben sich die Bromsalze, Bromglidine, Bromural, Veronal, Arsen, Atoxyl u. a. bewährt, auch gymnastische Uebungen, Hassage, hydrotherapeutische Anwendungen. Früher oder später wird die Unterbringung in einer Klinik oder einem Krankenhaus erforderlich, schon wegen der fortschreitenden geistigen Schwäche, der zeitweiligen Erregtheit und eventueller Selbstmordversuche. "Wenn wir sehen“, mit diesen Worten Kösters möchte ich meine Ausfübrungen schliessen, „wie Kranke in vorgeschrittenen Stadien einer Chorea Huntington mehr oder minder geistesschwach Jahre in einem gepolsterten Kastenbett in unausgesetzter maximaler Unruhe des ganzen Körpers verbringen, empfinden wir mit Schmerzen die Ohnmacht unserer Therapie".

\section{Literaturverzeichnis.}

1. Bonfigli, The journal of ment. pathol. No. 2. 1907. Cit. n. d. Bericht über die psych. Literatur 1907 (Snell). p. 218.

2. Curschmann, Deutsche Zeitschr. f. Nervenbeilk. XXXV. 3. u. 4. 1908. (zit. n. Schmidts Jahrb. Bd. 301.)

3. Engelen, Deutsche med. Wochenschr. 1907. Nr. 13.

1) Bezüglich der patholog. Anatomie verweise ich noch auf die - nach Abschluss dieses Beitrages - erschienene, sehr ausfübrliche Arbeit von Raecke, Beitrag z. path. Anat. der Hunt. Chorea chron. progr. hered. (Arch. f: Psych. 46. Bd. 2. H. 1909.) 
Ein Beitrag zum Krankheitsbild der Chorea chronica progressiva. 805

4. Facklam, Arch. f. Psych. 1898. 30. Bd.

5. Frank, Wiener klin. Wochenschr. 1904. Nr. 9.

6. Friedenthal, Petersb. med. Wochenschr. 1908. Nr. 29.

7. Heilbronger, Arcb. f. Psych. 1903. Bd. 36.

8. Hoffmann, Deutsche med. Wochenschr. 1909. Nr. 32.

9. Köster, Deutsche med. Wochenschr. 1909. Nr. 1.

10. Lebram, Deutsche med. Wochenschr. 1907. S. 2198.

11. Meyer, Deutsche med. Wochenschr. 1907. S. 2198.

12. Nerlich, Aerztl. Sachverst.-Zeitung. 1905. Nr. 3.

13. Ant. d'Ormoa, Rif. med. XX. 12. 1904. Zit. nach Schmidts Jahrb. 1905. Bd. 286.

14. Oppenheim, Lehrb. d. Nervenkrankh. 1902.

14a. Oppenteim und Hoppe, Arch. f. Psych. XXIV. S. 617.

15. Schabad, Deutsche med. Wochenschr. 1909. Nr. 22.

16. Schulz, Charité-Annalen. XXXII. 1908. Zit. nach Schmidts Jahrb. Bd. 301.

17. Skoczynski, Berl. klin. Wochenschr. 1902. Nr. 12 .

18. Steyerthal, Arch. f. Psych. 1908. 44. Bd.

19. Stier, Deutsche med. Wochenschr. 1902. Vereinsbeil. S. 167.

20. Strümpell, Lehrb. d. spez. Pathol. u. Therap. 10. Aufl.

21. Wallenberg, Deutsche med. Wochenschr. 1907. Nr. 52.

22. Weyrauch, Münchner med. Wochenschr. 1905. Nr. 6 .

23. Westphal, Deutsche med. Wochenschr. 1902. Nr. 4. 